\title{
Objectivité versus subjectivité dans l'évaluation des acquis des étudiants
}

\section{Marc Romainville}

\section{(2) OpenEdition}

Édition électronique

URL : http://journals.openedition.org/ripes/499

ISSN : 2076-8427

Éditeur

Association internationale de pédagogie universitaire

Référence électronique

Marc Romainville, «Objectivité versus subjectivité dans l'évaluation des acquis des étudiants », Revue internationale de pédagogie de l'enseignement supérieur [En ligne], 27(2) | 2011, mis en ligne le 16 janvier 2012, consulté le 07 septembre 2020. URL : http://journals.openedition.org/ripes/499

Ce document a été généré automatiquement le 7 septembre 2020.

Article L.111-1 du Code de la propriété intellectuelle. 


\title{
Objectivité versus subjectivité dans l'évaluation des acquis des étudiants
}

\author{
Marc Romainville
}

\section{Objectivité et subjectivité dans l'évaluation}

1 L'objectivité est définie de manière générale comme étant « la qualité de ce qui existe en soi, indépendamment du sujet pensant » (Trésor de la langue française). À l'inverse, la subjectivité est la « qualité de ce qui appartient seulement au sujet pensant ». Dans le domaine de l'évaluation des acquis des étudiants, on se rend alors vite compte que l'objectivité absolue est impossible, pour des raisons que je développerai ci-dessous, mais dont la principale est que les acquis des étudiants n'existent pas en eux-mêmes, indépendamment du regard que porte sur eux l'évaluateur. Cependant, on s'accordera tout autant à dire que la subjectivité la plus débridée constituerait un problème, puisqu'elle reviendrait à accepter que les résultats de l'évaluation et en particulier la décision de réussite ou d'échec de l'étudiant dépendent en définitive plus de l'évaluateur et de sa partialité que des connaissances et compétences de l'étudiant évalué.

2 Afin d'expliquer pourquoi l'objectivité est une chimère dans le domaine de l'évaluation des acquis, mais aussi pourquoi il convient d'encadrer et d'objectiver la subjectivité consubstantielle à cet acte, il faut en revenir à la définition de base de l'évaluation. Si l'on se réfère à la définition classique de Stufflebeam (Madaus, Scriven \& Stufflebeam, 1986) reprise ensuite par de très nombreux auteurs, évaluer consiste à mesurer puis à apprécier, à l'aide de critères, l'atteinte des objectifs d'enseignement, en trois étapes. La première étape réside dans le recueil systématique, valide et fidèle d'informations appropriées aux objectifs d'enseignement. C'est la phase d'observation et/ou de recueil de données. La deuxième étape consiste à interpréter les informations recueillies à l'aide de critères. C'est la phase d'analyse. Celle-ci débouche sur la troisième étape, à savoir l'établissement de conclusions et/ou la prise de décisions. C'est la phase du jugement à proprement parler. 
3 Ainsi, lorsqu'un enseignant souhaite évaluer les acquis de ses étudiants à l'issue d'un enseignement, il procède d'abord à un recueil standardisé d'informations. Par exemple, il soumet, à une même date et dans les mêmes conditions (temps de réponse standardisé, questions identiques ou équivalentes), tous les étudiants à un examen écrit durant lequel ces derniers produisent des réponses à des questions, censées mobiliser de manière représentative les connaissances et compétences qu'ils avaient à acquérir grâce à l'enseignement. Mais ces informations, les réponses des étudiants, ne parlent pas d'elles-mêmes. Encore faut-il les analyser à l'aide de critères, ces derniers étant en principe induits mécaniquement des objectifs. Par exemple, si l'enseignant vise à développer, dans un cursus de formation des maîtres, la compétence à analyser une situation éducative à l'aide de concepts psychopédagogiques, une question significative consiste à présenter aux étudiants une courte situation de classe en leur demandant d'identifier le(s) concept(s) dont la mobilisation serait utile à une meilleure lecture de cette situation. Lors de la correction, les productions des étudiants seront analysées selon un certain nombre de critères congruents par rapport aux objectifs : le concept mobilisé est-il adéquat à la situation ? L'étudiant se montre-t-il capable d'identifier explicitement les relations entre des éléments de la situation et le concept? Même analysées de manière rigoureuse en regard de critères, les productions des étudiants n'aboutissent pas non plus mécaniquement à un jugement ou une décision finale. Il faudra encore, dans un troisième temps, que l'enseignant construise un arbre de décisions, explicite ou implicite, lui permettant d'établir un jugement final (par exemple, la compétence est acquise ou non) à partir de ses analyses.

On le voit à travers cet exemple, évaluer n'équivaut pas à enregistrer de manière externe et objective des acquis, comme le thermomètre indique la température, sans l'intervention de l'homme (du moins postérieure à la fabrication de l'instrument). Au contraire, évaluer des acquis revient à construire un point de vue sur des performances censées représenter des acquis. Toute opération d'évaluation dépend donc du « sujet pensant ", à chacune de ses étapes d'ailleurs. La subjectivité ou l'intervention du « sujet pensant » intervient en effet aux trois étapes de l'évaluation décrites ci-dessus. Dans la première, le choix des informations à recueillir est déjà subjectif puisqu'il dépend des objectifs que s'est assigné l'enseignant. Or, ces objectifs peuvent être très différents pour un même enseignement inséré dans un même programme, en fonction des intentions, des intérêts et des conceptions des enseignants notamment. L'article de Kohler, Platteaux et Blons-Pierre publié dans ce numéro est éclairant à ce sujet puisqu'il montre que pour un même outil d'évaluation (un test de classement) diverses modélisations des compétences sous-jacentes sont possibles, induisant chacune une configuration particulière et donc subjective de l'outil.

Dans la deuxième phase, la subjectivité est tout aussi présente dans le choix des dimensions selon lesquelles les productions des étudiants seront analysées. D'autant que certaines de ces dimensions ne seront en fait pas le résultat d'un choix délibéré et conscient. On sait, en effet, que le regard porté par l'évaluateur sur une production peut être biaisé par quantité de variables a priori étrangères à ce que l'on cherche explicitement à mesurer; ce sont les fameux biais de l'évaluation qui en ruinent fréquemment la validité (cf. ci-dessous). La subjectivité n'est enfin pas absente de la troisième étape, puisque l'on sait que des analyses quasi identiques de performances d'étudiants peuvent aboutir à des conclusions diamétralement opposées (cf. la recherche de Johnston (1997) sur l'évaluation de thèse évoquée ci-dessous). La 
construction du jugement final ou l'élaboration de la décision de synthèse fait donc aussi appel à la subjectivité de l'évaluateur.

\section{Quelques données empiriques sur l'enseignement supérieur}

6 Le déficit d'objectivité de l'évaluation des acquis dans l'enseignement supérieur a été mis en évidence par une série d'études réalisées au sein du paradigme de la " docimologie », portant sur la fidélité et la validité des outils d'évaluation. Pour rappel, c'est Piéron qui a proposé, au début des années 1960, le terme de " docimologie » pour désigner un nouveau champ de recherche, appelé (avec l'assurance et selon le positivisme de l'époque...) «la science des examens». Essentiellement critique à l'origine, cette discipline a identifié une série de dysfonctionnements de l'évaluation (pour une synthèse de ces critiques, De Landsheere, 1980). Elle s'est ensuite orientée vers l'étude du processus de jugement des évaluateurs (Noizet \& Caverni, 1978).

7 Pour la docimologie, l'évaluation des acquis souffre de deux défauts majeurs. D'une part, sa fidélité n'est pas toujours assurée: des études empiriques ont montré que l'évaluation, quelle que soit la discipline, n'aboutit pas à des résultats similaires si elle est reproduite à un autre moment ou si elle est réalisée par une autre personne. D'autre part, la validité de l'évaluation a également fait l'objet de critiques : l'évaluation devrait mesurer ce qu'elle prétend mesurer et rien que ce qu'elle prétend mesurer. Une évaluation valide devrait donc s'en tenir à vérifier si les objectifs de l'enseignement ont été atteints par ceux à qui il était destiné. Elle ne devrait mesurer que cela et le mesurer de manière représentative. Or, les outils d'évaluation mesurent parfois des compétences qui n'ont pas fait l'objet d'un enseignement et, de plus, l'évaluateur établit son jugement sur la base d'autres critères que la seule performance " objective » de l'évalué. Citons quelques biais particulièrement étudiés: la contagion des évaluations (guidé par la recherche d'une consonance cognitive dans le jugement porté sur les individus, l'évaluateur se laisse influencer par le résultat d'évaluations antérieures); l'effet de halo (l'évaluateur est influencé par la connaissance qu'il peut avoir de caractéristiques externes à la cible de l'évaluation [niveau antérieur, origine socioculturelle et ethnique...]) ; effet d'ordre et de position dans le jugement des productions des évalués ...

8 Les relations entre ces deux qualités des outils d'évaluation mises en avant par la docimologie et la tension entre l'objectivité et la subjectivité sont multiples. D'une part, une évaluation parfaitement fidèle serait par la même occasion objective puisqu'elle ne dépendrait plus de la diversité des «sujets pensants ». D'autre part, la validité d'une évaluation participe aussi à son objectivité puisqu'elle la fait porter sur un objet correctement délimité (l'atteinte des objectifs d'enseignement), qui dépend dès lors moins de la variabilité des biais avec lesquels les « sujets pensants » appréhenderont les productions des étudiants.

Des études de docimologie, parfois anciennes, ont été spécifiquement réalisées dans l'enseignement supérieur, avec des résultats proches de ceux qui ont été engrangés pour l'enseignement primaire et secondaire (Ashcroft \& Palacio, 1996; Beattie, 1995; Brown \& Glasner, 1999 ; Heywood, 2000 ; Warren Piper, 1994). Nous n'en évoquerons ici 
que quelques-unes à titre d'illustration, un relevé plus complet étant disponible par ailleurs (Romainville, 2002).

10 Ainsi, dès les années 1930, une série d'études ont été consacrées à la fidélité des examens dans l'enseignement supérieur: des correcteurs ont été invités à noter à nouveau des copies qu'ils avaient corrigées antérieurement. Les notations d'une centaine de professeurs de mathématiques ont été comparées à propos de cinq mêmes copies. Les résultats sont sans appel: dans la détermination de la note, la part du correcteur se révèle finalement plus décisive que celle de la performance réalisée par l'étudiant.

11 Ces premières études ont été confirmées par un programme de recherche de la Society for Research into Higher Education réalisé dans les années 1960, puis par des études portant plus spécifiquement sur des disciplines particulières, notamment les sciences médicales et les sciences de l'ingénieur. Dans une étude de Warren Piper (1994) sur les examinateurs externes des universités anglaises, ceux-ci s'interrogent régulièrement sur la fidélité de l'évaluation. Ils se déclarent parfois surpris de la diversité des notes en cas d'examinateurs multiples. Ils s'interrogent aussi sur la comparabilité des jugements évaluatifs dans le temps et entre les filières : comment garantir, par exemple, qu'un diplôme sanctionne un même niveau d'études d'année en année ?

12 Une des explications du manque de fidélité réside dans le fait que les critères d'évaluation varient considérablement d'un examinateur à l'autre, parce que ces examinateurs sont guidés par des cadres de référence différents pour juger leurs étudiants (Warren Piper, 1994 ; Webster, Pepper \& Jenkins, 2000). Par exemple, certains correcteurs tiennent compte de la performance des autres étudiants, d'autres s'attachent à repérer les signes d'un progrès depuis le début du cours. Autre exemple, le jugement porté sur une dissertation dépend de ce que le correcteur valorise dans un tel travail: certains privilégient l'apport de données empiriques, d'autres l'aspect théorique. Pour une même filière, les jurys de différents établissements n'attribuent pas les mentions selon les mêmes critères et sont ignorants des critères appliqués ailleurs. Par exemple, la mention la plus élevée n'est accordée par certains examinateurs qu'à la condition que le candidat se distingue par son originalité; d'autres réservent cette mention au cas où le candidat fait montre de compétences de communication exceptionnelles.

13 Même quand les correcteurs se réfèrent à des critères identiques, ils ne leur attribuent pas toujours la même signification et ils ne les font pas intervenir selon la même pondération, ce qui explique que des commentaires identiques ou proches apparaissent sur des copies ayant obtenu des notes très différentes ou que des notes identiques soient justifiées par des commentaires antinomiques. Ainsi, si les enseignants sont assez unanimes pour identifier les critères pertinents pour classer les étudiants (l'analyse critique, par exemple), on observe que des comportements précis très différents sont retenus comme indicateurs de ce critère selon la discipline enseignée et même parfois selon les théories privilégiées au sein de la même discipline (Ecclestone, 2001). Dans le même sens, Webster (2000) a demandé à 24 enseignants de préciser ce qu'ils entendaient quand ils annonçaient à leurs étudiants qu'un de leurs critères d'évaluation résiderait dans la "qualité de l'analyse». Pour un premier groupe d'enseignants, l'analyse est définie comme la décomposition d'un tout en ses différentes parties, de manière à mieux comprendre les relations qui les unissent. Pour un second groupe, il s'agit de l'application d'outils méthodologiques aux données 
recueillies. Neuf autres des 24 enseignants interrogés fournissent chacun une définition qui ne se rapproche ni de celle du premier groupe ni de celle du second. La même étude révèle que les critères effectivement pris en compte lors de la correction diffèrent parfois considérablement des critères annoncés. Ainsi, tous les critères annoncés ne sont pas nécessairement appliqués et certains critères effectifs, induits à partir des annotations des copies, n'avaient pas été annoncés.

Plusieurs études montrent que des problèmes de fidélité se rencontrent aussi pour l'obtention du diplôme de doctorat. Johnston (1997) a ainsi réalisé une analyse détaillée de rapports de thèse. Sur les 16 thèses analysées et jugées chacune par quatre professeurs, trois d'entre elles font l'objet de désaccord entre les enseignants à propos de la décision d'échec ou de réussite. Pour l'une des thèses analysées, un rapport relève la qualité et l'originalité de la revue de la littérature ("éclectique, organisée et cohérente »), alors que le rapport de l'expert prônant l'échec contient sept pages de critiques sur cette partie de la thèse, qui se terminent en regrettant que le candidat n'ait pas eu recours à la structure classique et académique d'un relevé de la littérature. Dans un autre cas, c'est manifestement une incompatibilité idéologique qui explique le désaccord. Des commentaires identiques n'ont, de plus, pas le même impact chez tous les rapporteurs: des suggestions de modifications de forme peuvent justifier un avis d'acceptation sous condition de modification, tout comme elles peuvent être simplement mentionnées après un avis favorable sans condition. En ce qui concerne le type de critères utilisés pour juger de la qualité de la thèse, les rapporteurs se réfèrent tous à des critères de qualité formelle (écriture et présentation), mais divergent quant aux autres critères (gestion de la complexité, originalité, pensée critique, caractère publiable du travail...), au point que peu de thèmes sont communs à l'ensemble des rapports. Enfin, un même critère peut revêtir des sens différents: ainsi, quand les rapporteurs se réfèrent au caractère " publiable » du travail de recherche, cela signifie, pour certains, que la thèse est publiable telle quelle; pour d'autres, que le travail mérite publication, mais après avoir subi une série de révisions parfois importantes. Dans le même sens, Morley, Leonard et David (2002) ont décrit l'importante hétérogénéité des pratiques d'évaluation de la thèse de doctorat selon les institutions, les facultés et les départements : poids relatif de l'oral par rapport à l'écrit, modalités de rapports de thèse et de constitution du jury.

La validité de l'évaluation des acquis dans l'enseignement supérieur a aussi fait l'objet de questionnements critiques. On a ainsi mis en évidence l'existence de biais idéologiques dans la correction de copies. À titre d'exemple, l'étude de Husbands (1976) a porté sur un département de sciences sociales au sein duquel deux écoles de pensée coexistaient, l'une centrée sur l'action sociale et l'autre sur des approches sociales cliniques. Six enseignants, dont on connaissait le « penchant » épistémologique, ont été invités à noter des copies d'étudiants, la moitié de ces copies manifestant une certaine sympathie pour la première école de pensée et l'autre moitié pour la seconde. L'existence de biais idéologiques n'est pas systématique : certains correcteurs semblent faire fi de leurs options méthodologiques personnelles. Mais d'autres surévaluent assez systématiquement les copies valorisant les cadres d'analyse qu'ils privilégient euxmêmes en tant que chercheurs. L'auteur conclut son étude en montrant comment l'attitude stratégique de certains étudiants qui adaptent leur discours aux positions épistémologiques qu'ils pensent être celles de leur correcteur est finalement assez rationnelle. 
16 L'existence de biais culturels dans les procédures d'évaluation des étudiants a aussi fait l'objet d'investigation, dans le contexte d'un enseignement supérieur mondialisé. On s'est notamment interrogé sur l'adéquation de certaines formes d'examen aux différents publics d'étudiants, en particulier les étudiants étrangers et ceux qui appartiennent aux minorités ethniques ou culturelles. Ainsi, De Vita (2002) a montré que les différentes formes d'examen n'offrent pas aux étudiants d'origine culturelle différente les mêmes opportunités de manifester leur maîtrise du cours. Par exemple, les étudiants d'origine chinoise inscrits dans les universités anglaises semblent, par certains côtés, désavantagés par les QCM car ils répugnent à choisir au hasard une réponse en cas de doute. Par d'autres aspects cependant, les examens écrits à réponse longue les désavantagent également, comme tous les étudiants non anglophones d'ailleurs, tant ce type d'examen mesure autant (sinon davantage) les compétences langagières que la stricte maîtrise des connaissances.

17 Un autre aspect de la validité de l'évaluation des acquis qui est de plus en plus souvent étudié est celui de la nécessaire congruence entre le dispositif d'évaluation et la logique de formation. En effet, une évaluation valide est aussi celle qui recourt à des instruments de mesure compatibles avec les dispositifs de formation. En particulier, le problème de l'évaluation des compétences fait actuellement l'objet de nombreux travaux, tant cette forme d'évaluation pose de redoutables et spécifiques questions docimologiques (cf. par exemple, Gérard, 2008). On s'aperçoit notamment qu'une authentique approche par compétences bouleverse nécessairement les pratiques d'évaluation des acquis. Celles-ci évoluent vers des approches davantage intégrées et interdisciplinaires; globales, continues et formatives; authentiques et en contexte parce qu'une compétence est bien souvent interdisciplinaire et globale et parce qu'elle se développe de manière progressive et qu'elle ne peut s'appréhender qu'en situation. De ce point de vue, une pratique évaluative utilisée également comme outil de développement de compétences abolit en quelque sorte les anciennes frontières entre formation et évaluation.

\section{Encadrer et objectiver la subjectivité}

18 Éradiquer toute subjectivité dans l'évaluation des acquis ne semble en définitive de l'ordre ni du possible ni du souhaitable. Les études évoquées ci-dessus montrent d'abord que la quête d'une objectivité absolue relève de la chimère. Cette quête nous entraînerait indéfiniment vers un travail de précision et d'explicitation de plus en plus minutieux, sans que l'on ait jamais l'assurance qu'il aboutisse à évacuer totalement le sujet de l'acte d'évaluation. L'article de Cordonnier dans ce même numéro nous met d'ailleurs bien en garde contre les errements et la déresponsabilisation auxquels une quête excessive de l'objectivité pourrait conduire. Même lorsque les évaluateurs se sont mis d'accord sur des critères, l'appréciation de ceux-ci réintroduit inévitablement une part de subjectivité. L'étude de Berthiaume, David et David rapportée également dans ce numéro montre ainsi que même une grille critériée précise et opérationnelle contient des zones de flou et requiert donc des interprétations subjectives, par exemple en matière de spécification des seuils de réussite. De surcroît, surtout dans l'enseignement supérieur, la multiplicité des points de vue en ce qui concerne les acquis des étudiants constitue une richesse, qui pourrait être mise à mal par un trop grand souci de standardiser les pratiques sous prétexte de les rendre objectives. 
La subjectivité n'est donc pas à éradiquer, mais à encadrer et à objectiver. Encadrer la subjectivité signifie lui imposer des limites et développer des procédures garantissant qu'elle se déploie dans des proportions acceptables. Objectiver la subjectivité revient à tout à la fois à reconnaître aux « sujets pensants » évaluateurs la légitimité d'un point de vue spécifique, mais à réclamer aussi que ce point de vue ait été l'objet d'une explicitation préalable auprès des étudiants, de sorte que l'évaluation ne s'apparente pas au « jeu du chat et de la souris ».

Les manières d'encadrer et d'objectiver la subjectivité sont multiples et diverses. La plus fondamentale est sans doute que chaque enseignant, dans le respect de sa liberté académique, explicite ses objectifs d'enseignement et les communique de manière claire et transparente à ses étudiants. En aval de cette opération et en lien avec elle, l'explicitation des procédures, modalités et critères d'évaluation participe aussi à l'objectivation d'une partie du travail subjectif qui sera celui de l'évaluateur (cf. l'expérience d'élaboration de grille critériée rapportée dans ce numéro).

21 Une autre façon de réduire la subjectivité réside dans le développement de l'intersubjectivité. C'est en effet en prenant conscience de la diversité des points de vue au sein d'un groupe que l'on peut mieux identifier le sien propre. Des regards évaluatifs pluriels, comme dans la co-correction ou dans l'évaluation collective telle qu'elle est présentée dans l'article de Clerc et Daniel dans ce numéro, permettent de prendre conscience de la spécificité et donc de la subjectivité de son point de vue.

La tension entre objectivité et subjectivité restera donc inhérente à tout travail d'évaluation des acquis. En cette matière comme en tant d'autres, le mieux est l'ennemi du bien. Autrement dit, se fixer comme objectif d'éradiquer totalement la subjectivité est non seulement illusoire, mais risque de produire des effets néfastes non négligeables, comme l'atomisation de l'évaluation ou la déresponsabilisation de ses acteurs. Mais il serait tout aussi fallacieux de prétendre que la subjectivité, étant donnée sa consubstantialité à tout acte d'évaluation, est à accepter telle quelle et à laisser se déployer sans garde-fou. L'ensemble des textes réunis dans ce numéro montre bien que toutes les équipes pédagogiques sont à la recherche d'un compromis équilibré et acceptable en cette matière et que plusieurs voies peuvent y mener : l'établissement de grilles critériées, l'explicitation des modèles sous-jacents aux outils d'évaluation, la co-évaluation ... La voie principale et commune à l'ensemble des expériences rassemblées ici réside sans conteste dans un souci d'explicitation et d'analyse critique de ses propres choix d'évaluateur. Tout comme la prise de conscience est le début de la sagesse, l'explicitation raisonnée et critique de sa propre subjectivité constitue sans nul doute une des meilleures voies vers davantage d'objectivité.

\section{BIBLIOGRAPHIE}

Ashcroft, K., \& Palacio, D. (1996). Researching into assessment and evaluation in colleges and universities. London : Kogan Page. 
Beattie, J.-F. (1995). Évaluation dans l'enseignement supérieur. Gestion de l'enseignement supérieur, 7(3), 307-324.

Brown, S. \& Glasner, A. (Eds) (1999). Assessment matters in higher education. London : Open University Press.

De Landsheere, G. (1980). Évaluation continue et examens. Précis de docimologie. Bruxelles : Éditions Labor.

De Vita, G. (2002). Cultural equivalence in the assessment of home and international business management students. Studies in Higher Education, 27(2), 221-231.

Ecclestone, K. (2001). «I know a 2:1 when I see it » : understanding criteria for degree classifications in frenchised university programmes. Journal of Further and Higher Education, 25(3), 301-313.

Gérard, F.-M. (2008). Évaluer des compétences. Bruxelles : De Boeck.

Heywood, J. (2000). Assessment in Higher Education. London: Jessica Kingsley Publishers.

Husbands, C. (1976). Ideological bias in the marking of examinations. Research in higher education, 15, 17-38.

Johnston, S. (1997). Examining the examiners: an analysis of examiners' reports on doctoral thesis. Studies in Higher Education, 22 (3), 333-347.

Madaus, G. F., Scriven, M., \& Stufflebeam, D. L. (1986). Evaluation models. Viewpoints on educational and human services evaluation. Hingham: Kluwer Academic Publishers.

Morley, L., Leonard, D., \& David, M. (2002). Variations in vivas: quality and equality in British $\mathrm{PhD}$ assessments. Studies in higher education, 27(3), 263-274.

Noizet, G., \& Caverni, J.-P. (1978). Psychologie de l'évaluation scolaire. Paris : Presses Universitaires de France.

Romainville, M. (2002). L'évaluation des acquis des étudiants dans l'enseignement universitaire. Paris : Rapport établi à la demande du Haut Conseil de l'évaluation de l'école, disponible sur le site : http://www.hce.education.fr/index.php

Warren Piper, D. (1994). Are professors professional ? The Organisation of University Examinations. London: Jessica Kingsley Publishers.

Webster, F., Pepper, D., \& Jenkins, A. (2000). Assessing the undergraduate dissertation. Assessment and evaluation in Higher education, 25(1), 71-80.

\section{RÉSUMÉS}

Il est fréquent d'entendre regretter que l'évaluation des acquis des étudiants manque d'objectivité. Un bon nombre de pratiques évaluatives innovantes vise d'ailleurs spécifiquement à restreindre la subjectivité des évaluations. Mais que faut-il entendre par là ? L'évaluation peutelle vraiment être objective ? La subjectivité n'est-elle pas consubstantielle à tout acte évaluatif ? L'objectif du présent article est de discuter de cette tension entre subjectivité et objectivité dans l'évaluation des acquis en trois temps. Dans une première partie, on cherchera à définir ce que recouvrent les notions d'objectivité et de subjectivité dans le champ de l'évaluation des acquis. Quelques données empiriques se rapportant à cette tension dans l'enseignement supérieur seront présentées dans la deuxième partie. On tentera enfin, dans la troisième partie, de délimiter les 
contours d'un compromis à trouver entre une recherche chimérique d'une objectivité parfaite et les dérives non moins redoutables d'une subjectivité débridée.

INDEX

Mots-clés : évaluation des acquis, objectivité, pratique évaluative, subjectivité

\section{AUTEUR}

\section{MARC ROMAINVILLE}

Facultés universitaires de Namur (Belgique)

marc.romainville@fundp.ac.be 